\title{
A COMPARATIVE STUDY OF PULMONARY AND EXTRA- PULMONARY TUBERCULOSIS IN BHUTAN (2015-2017)
}

\author{
Zangpo $\mathrm{T}^{1}$, Tshering $\mathrm{C}^{2}$, Tenzin $\mathrm{P}^{3}$, Rinzin, $\mathrm{C}^{3}$, Dorji $\mathrm{C}^{4}$,Nima $\mathrm{G}^{5}$, Tenzing $\mathrm{P}^{6}$, Tsheten $\mathrm{T}^{7}$ \\ ${ }^{1}$ Dechencholing BHU-I, Ministry of Health, Thimphu, Bhutan \\ ${ }^{2}$ Deothang Military Hospital, Samdrupjongkhar, Bhutan \\ ${ }^{3}$ National Tuberculosis Control Program, DoPH, Ministry of Health, Thimphu, Bhutan \\ ${ }^{4}$ Gidakom Hospital, Thimphu, Bhutan \\ ${ }^{5}$ Jigme Dorji National Referral Hospital, Thimphu, Bhutan \\ ${ }^{6}$ Lungtenphu Military Hospital, Thimphu, Bhutan \\ ${ }^{7}$ Royal Centre for Disease Control, Serbithang, Thimphu, Bhutan
}

\begin{abstract}
Introduction: Extrapulmonary Tuberculosis (EPTB) has been increasingly diagnosed and reported compared to Pulmonary TB (PTB) in Bhutan in the recent years. In this comparative study, our study describes the epidemiology, diagnostic modalities used, inconsistency in case-classification and treatment outcomes of PTB and EPTB cases diagnosed from 2015-2017.
\end{abstract}

Methods: A retrospective descriptive study was conducted by retrieving patient records maintained at the 11 TB Reporting Centres. Using two stage cluster sampling technique, nine primary sampling units (9-PSUs) were generated for the years 2015, 2016 and 2017 respectively. Based on the highest caseloads among 32 TB Reporting Centres, nine primary clusters were selected first and final sample of 350 for each year were drawn using systematic random sampling technique from the PSUs.

Results: We recruited a total of 1048 cases (PTB=555, EPTB=493) in the final descriptive analysis. The median age of the subjects was 27 years (range 1-87) with slight female predominance (53\%). Students were the highest $(23.85 \%)$ followed by farmers (17.94\%). The proportion of PTB was (52.95\%), EPTB of $(47.08 \%)$ and clinically diagnosed EPTB accounted for (92\%), which is extremely high. Twelve miliary/disseminated TB cases (1.15\%) were miss-classified as EPTB and (56\%) EPTB cases were not classified based on the body sites involved. The overall treatment success rate (TSR) of PTB and EPTB was $(93.26 \%)$.

Conclusion: We recommend an expert team to revisit National TB treatment guidelines on diagnosis of EPTB and ensure EPTB site/s are properly identified and documented. Sensitize clinicians and other units (ENT, Surgery, Orthopedics, MCH, General OPD etc.) on the referral of EPTB cases to Medical Specialist/Chest Physician for consultation.

Key words: Extra-pulmonary tuberculosis, Pulmonary tuberculosis, Descriptive study, Bhutan

\section{INTRODUCTION}

Tuberculosis is an infectious disease caused by the bacillus Mycobacterium tuberculosis, typically

Correspondence:
Dr. Tadin Zangpo
MBBS, MPH (Biosecurity) \& One Health Epidemiology Fellowship
Medical Officer \& Epidemiologist
Dechencholing BHU-1, Health Sector, Thimphu District
Administration
Thimphu, Bhutan
E-mail: zheynuapa@gmail.com
Mobile: + 975-17828860

affects lungs (PTB) and it can also affect other body sites (EPTB). The disease is spread through respiratory droplet infection when the infected patient coughs, sneezes or spits. It is estimated that a small proportion $(5-15 \%)$ of the estimated 1.7 billion people infected with $M$. tuberculosis could develop TB during their lifetime. In general, the probability of developing TB is higher among people affected with HIVIAIDS, under-nutrition, diabetes, smoking and alcohol consumption ${ }^{(1)}$.

According to the World Health Organization (WHO), tuberculosis falls in one of the top 10 
causes of global death. About 10.4 million people has fallen sick with TB, and 1.7 million have died from TB (including 0.4 million among people with HIV) in 2016 with over $95 \%$ of TB deaths occurring in low and middle income counters ${ }^{(2)}$.

The National Tuberculosis Control Programme (NTCP) in the Ministry of Health in Bhutan was established in 1986. In 2001 the proportion of EPTB of all the TB patients was $26 \%$. In 2010 the proportion of EPTB has increased to $39 \%$ and an alarming increase was seen by the end of 2016 , wherein almost half $(49 \%)$ of all TB cases registered were classified as EPTB $\left.{ }^{(3,}{ }^{4}\right) \mathrm{DoPH}$, Ministry of Health.

The availability of computerized tomographic scan (CT-scan), magnetic resonance imaging, laparoscopy, endoscopy and aspiration biopsy have tremendously helped in anatomical localization and sampling of EPTB sites in diagnostic confirmation (5).

As EPTB occupying about half of the tuberculosis infection proportion in Bhutan is a cause of concern and at the same time a huge economic burden for a small developing country like ours. We conducted this comparative study on PTB and EPTB to obtain baseline information on TB epidemiology, report on any miss-classification and understand TB treatment outcomes. In Bhutan we have a total 32 TB Reporting Centers with at least one each for a district and some bigger districts like Chukha, Samtse and Trashigang has two to three TB Reporting Centres ${ }^{(4)}$.

\section{METHODOLOGY}

A retrospective descriptive study was conducted by retrieving patient records maintained at the TB Reporting Centres sampled as clusters in our study for the years 2015, 2016 and 2017.

\section{Study period}

The descriptive study was conducted from March to June 2018.

\section{Sampling strategy}

Target population

The whole of the Bhutanese people were the target population for this study. As the study was conducted nationwide with samples across nine districts, the findings of this study could be extrapolated to the whole of the Bhutanese population.

\section{Source population}

All the PTB and EPTB cases registered at the 32 TB Reporting Centres formed the source population.

Study areas/sites

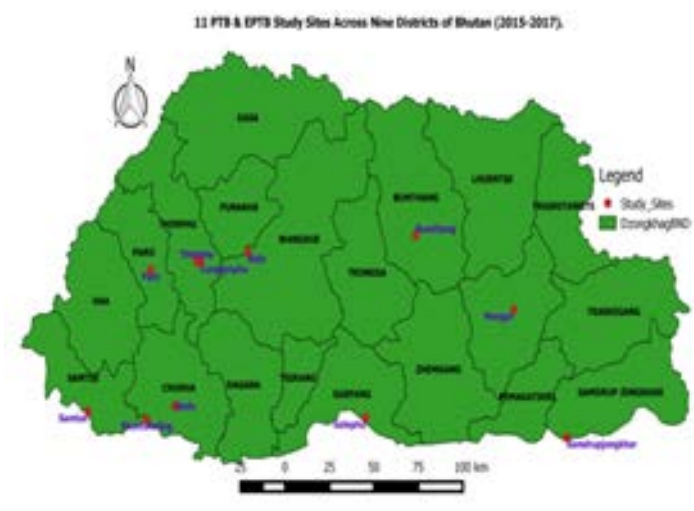

Figure 1. PTB and EPTB study sties, 2015-2017

We used two stage cluster sampling technique Nine primary sampling units (9-PSUs) were generated using OpenEpi Version 3 software for the years 2015, 2016 and 2017. The actual samples required for each year from the nine clusters were 288 . Based on the highest caseloads among the 32 TB Treatment Centres, nine primary clusters were selected first and final sample of 350 for each year were selected using systematic random sampling technique (SRS).

\section{Definition and diagnosis of PTB and EPTB}

PTB refers to any bacteriologically confirmed or clinically diagnosed case of TB that involved the lung parenchyma or trachea-bronchial tree with or without the involvement of any other organs in the body. Any bacteriologically confirmed or clinically diagnosed cases of TB involving other organs other than the lungs e.g. pleura, lymph nodes, abdomen, genitourinary track, skin, joints and bones, meninges, tuberculous pleural effusion without radiographic abnormalities in the lung parenchyma constitutes a case of EPTB ${ }^{(6)}$.

\section{Data collection and management}

Data on patient demography, mode of diagnosis, TB case classification, sputum examination and 
follow up, voluntary counselling and testing (VCT) and treatment outcomes were retrieved from the TB register, TB treatment card and Tuberculosis Surveillance and Information System (TbISS) online database system. Wherever feasible, we have contacted individual patients, for whom the data were incomplete in the above records maintained. Data base was built and entered in Epilnfo 7 . STATA version 14 was used for data analysis, QGIS version 2.12.2 software for generating maps and excel for graph preparation. Mainly, descriptive statistical analysis was performed to generate counts and percentages. Results are presented mostly in tables, graphs and using maps.

\section{Ethical Considerations}

The study was approved by Research Ethics Board of Health (REBH) in Bhutan under the Ministry of Health vide its approval letter reference. No. REBH/Approval/2017/096 31st May, 2018.

\section{RESULTS}

\section{Socio-demographic characteristics}

The study included a total of 1048 (PTB=555 and $E P T B=493$ ) subjects from a total of 11 health centers from 2015-2017. PTB and EPTB combined socio-demography characteristics showed that the median age of the study participants was 27 years, which ranged from one to 87 years. Females were slightly more than $(53 \%)$ than males. By occupation, students were the highest $(23.85 \%)$ followed by farmers (17.94\%). By ethnic group, Sharchop constituted the highest (48.09\%) followed by Ngalop (32.54\%) and (46.47\%) of our participants were married. While the participants in PTB and EPTB showed significant difference by age groups ( $p$ value $<0.05$ ), other variables such as gender, place of residence, occupation, qualification, ethnicity and marital status between PTB and EPTB did not show any significant difference ( $p$ value $>0.05$ ) (Table 1).

Table 1. Distribution of PTB and EPTB by demographic characteristics in Bhutan, 2015-2017.

\begin{tabular}{|c|c|c|c|c|}
\hline \multirow{2}{*}{\multicolumn{2}{|c|}{$\begin{array}{l}\text { Demographic characteristics } \\
\mathrm{n}(\%)\end{array}$}} & \multirow{2}{*}{\begin{tabular}{|c} 
PTB (n=555) \\
$\mathrm{n}(\%)$
\end{tabular}} & \multirow[t]{2}{*}{ EPTB $(n=493)$} & \multirow[b]{2}{*}{$p$-value } \\
\hline & & & & \\
\hline \multirow[t]{2}{*}{ Gender } & Female & $290(52.25)$ & 266 (53.96) & $0.581^{*}$ \\
\hline & Male & 265 (47.75) & $227(46.04)$ & \\
\hline \multirow[t]{4}{*}{ Age category } & $0-14$ & $14(2.52)$ & $30(6.09)$ & $0.035^{*}$ \\
\hline & $15-44$ & $428(77.12)$ & 367 (74.44) & \\
\hline & $45-64$ & $72(12.97)$ & 65 (13.18) & \\
\hline & $>65$ & $41(7.39)$ & $31(6.29)$ & \\
\hline \multirow[t]{3}{*}{ Residence } & Rural & $301(54.23)$ & 266 (53.96) & $0.223^{*}$ \\
\hline & Urban & $245(44.14)$ & $211(42.80)$ & \\
\hline & Missing & $9(1.62)$ & $16(3.25)$ & \\
\hline \multirow[t]{13}{*}{ Occupation } & Farmer & $108(19.46)$ & $80(16.23)$ & $0.878^{\#}$ \\
\hline & Home-maker & $73(13.15)$ & 67 (13.59) & \\
\hline & Civil Servant & $30(5.41)$ & $31(6.29)$ & \\
\hline & Corporate & $22(3.96)$ & $17(3.45)$ & \\
\hline & Military & $21(3.78)$ & $16(3.25)$ & \\
\hline & Students & $127(22.88)$ & $123(24.95)$ & \\
\hline & Child & $3(0.54)$ & $6(1.22)$ & \\
\hline & Monk & $13(2.34)$ & $7(1.42)$ & \\
\hline & Construction worker & $10(1.80)$ & $10(2.03)$ & \\
\hline & Private business & $67(12.07)$ & $58(11.76)$ & \\
\hline & Health worker & $4(0.72)$ & $6(1.22)$ & \\
\hline & National work force & $1(0.18)$ & $1(0.20)$ & \\
\hline & Mining & $1(0.18)$ & $0(0.00)$ & \\
\hline
\end{tabular}




\begin{tabular}{|c|c|c|c|c|}
\hline & Dependent & $22(3.96)$ & $14(2.84)$ & \\
\hline & Prisoner & $5(0.90)$ & $6(1.22)$ & \\
\hline & Missing & $48(8.65)$ & $51(10.34)$ & \\
\hline Qualification & No schooling & $152(27.39)$ & $123(24.95)$ & $0.144^{\#}$ \\
\hline & Non-formal education & $12(2.16)$ & $10(2.03)$ & \\
\hline & Primary school $(<=6)$ & $55(9.91)$ & $53(10.75)$ & \\
\hline & Lower secondary school & $56(10.09)$ & $32(6.49)$ & \\
\hline & Higher secondary school & 161(29.01) & $139(28.19)$ & \\
\hline & Degree and above & $45(8.11)$ & $54(10.95)$ & \\
\hline & Monastic & $12(2.16)$ & $8(1.62)$ & \\
\hline & Pre-school & $0(0.00)$ & $2(0.41)$ & \\
\hline & Missing & $62(11.17)$ & $72(14.60)$ & \\
\hline Ethnicity & Indian & $10(1.80)$ & $11(2.23)$ & $0.698^{*}$ \\
\hline & Sharchop & $273(49.19)$ & $231(46.86)$ & \\
\hline & Ngalong & $181(32.61)$ & $160(32.45)$ & \\
\hline & Lhotshampa & $90(16.22)$ & $88(17.85)$ & \\
\hline & Missing & $1(0.18)$ & $3(0.61)$ & \\
\hline Marital status & Unmarried & $211(38.02)$ & $203(41.18)$ & $0.29^{*}$ \\
\hline & Married & $267(48.11)$ & $220(44.62)$ & \\
\hline & Separated & $7(1.26)$ & $7(1.42)$ & \\
\hline & Widow & $18(3.24)$ & $8(1.62)$ & \\
\hline & Missing & $52(.37)$ & $55(11.16)$ & \\
\hline
\end{tabular}

*Pearson Chi-Square \#Fisher's Exact

\section{Mode of diagnosis of PTB and EPTB}

Through 2015 to $2017,98.66 \%$ of the PTB were diagnosed by smear microscopy. The next common bacteriological diagnosis was by culture $(91.96 \%)$, followed by Line Probe Assay (LPA) (72.54\%). About $85.05 \%$ of clinical diagnosed cases of PTB were based on X-ray findings. CT scan and Ultrasonography (USG) were minimally employed ( $7.47 \%$ and $1.87 \%$ respectively). EPTB were mostly diagnosed by histopathology on the basis of biopsy and fine needle aspiration cytology (FNAC - 82.48\%) (Table 2). 


\begin{tabular}{|c|c|c|c|c|}
\hline \multirow{2}{*}{ Mode of diagnosis } & \multirow{2}{*}{ Investigation types } & \multirow{2}{*}{ Total tested } & \multicolumn{2}{|c|}{ Positivity } \\
\hline & & & $\mathrm{n}$ & $\%$ \\
\hline \multicolumn{5}{|c|}{ PTB Diagnosis } \\
\hline \multirow{8}{*}{ Bacteriology } & Smear & 447 & 442 & 98.66 \\
\hline & Culture & 448 & 412 & 91.96 \\
\hline & GeneXpert & 446 & 184 & 41.07 \\
\hline & LPA & 447 & 325 & 72.54 \\
\hline & X-Ray & 447 & 269 & 60.04 \\
\hline & MRI & 448 & 5 & 1.12 \\
\hline & CT scan & 448 & 5 & 1.12 \\
\hline & USG & 448 & 1 & 0.22 \\
\hline \multirow{4}{*}{ Clinical } & X-Ray & 107 & 91 & 85.05 \\
\hline & MRI & 106 & 0 & 0 \\
\hline & CT scan & 107 & 8 & 7.48 \\
\hline & USG & 107 & 2 & 1.87 \\
\hline \multicolumn{5}{|c|}{ EPTB Diagnosis } \\
\hline \multirow{8}{*}{ Bacteriology } & Smear & 38 & 5 & 12.82 \\
\hline & Culture & 38 & 2 & 5.13 \\
\hline & GeneXpert & 38 & 4 & 10.26 \\
\hline & LPA & 38 & 0 & 0 \\
\hline & X-Ray & 38 & 10 & 25.64 \\
\hline & MRI & 38 & 2 & 5.13 \\
\hline & CT scan & 38 & 2 & 5.13 \\
\hline & USG & 38 & 1 & 2.56 \\
\hline \multirow{4}{*}{ Clinical } & X-Ray & 449 & 169 & 37.22 \\
\hline & MRI & 450 & 28 & 6.17 \\
\hline & CT scan & 450 & 49 & 10.79 \\
\hline & USG & 450 & 85 & 18.72 \\
\hline \multirow{2}{*}{ Histopathology } & FNAC & 137 & 113 & 82.48 \\
\hline & Biopsy & 134 & 30 & 21.9 \\
\hline
\end{tabular}

\section{Classification of PTB and EPTB}

The overall proportion of PTB was $52.95 \%$ (95\% Cl: $49.93 \%-55.98 \%$ ), while the proportion of EPTB was $47.08 \%$ (95\% Cl: $44.01 \%$ - 50.01\%) (Table 3). The proportion of EPTB clinically diagnosed accounted for $92.09 \%\left((440+14) / 493^{*} 100\right)$, which is extremely high percentage with no evidence of
M. tuberculosis. Bacteriologically confirmed EPTB was very low at $7.91 \%$. Similarly, the percentage of clinically diagnosed PTB was $19.28 \%$ and bacteriologically diagnosed PTB was $80.72 \%$ respectively. Relapsed cases $(n=66)$ accounted for the maximum number of previously treated TB cases with $(n=55,83 \%)$ followed by treatment after failure $(n=8,12.12 \%)$. 
Table 3. Classification of PTB and EPTB in Bhutan, 2015-2017.

\begin{tabular}{|c|c|c|c|c|c|}
\hline \multirow{2}{*}{\multicolumn{2}{|c|}{$\begin{array}{l}\text { TB type } \\
n\end{array}$}} & \multicolumn{2}{|c|}{ New } & \multicolumn{2}{|c|}{ Previously treated } \\
\hline & & \multirow{2}{*}{$\frac{\%}{401}$} & \multirow{2}{*}{$\begin{array}{l}n \\
89.51\end{array}$} & \multirow{2}{*}{$\frac{\%}{47}$} & \multirow[b]{2}{*}{10.49} \\
\hline \multirow{3}{*}{ PTB } & Pulmonary $\mathrm{BC}^{*}$ & & & & \\
\hline & Pulmonary $C D^{* *}$ & 104 & 97.20 & 3 & 2.80 \\
\hline & Overall PTB (\%) & \multicolumn{4}{|c|}{52.95 (95\% Cl: 49.93 - 55.98) } \\
\hline \multirow{3}{*}{ EPTB } & Extrapulmonary BC & 37 & 94.87 & 2 & 5.13 \\
\hline & Extrapulmonary CD & 440 & 96.92 & 14 & 3.08 \\
\hline & Overall EPTB (\%) & \multicolumn{4}{|c|}{$47.08 \%(95 \%$ Cl: $44.01-50.01)$} \\
\hline
\end{tabular}

\section{Classification of EPTB by body sites involvement}

The most common EPTB sites involved was pleural effusion (17.56\%), followed by lymphatic (13.17\%) and gastrointestinal system (5.06\%). More than half of the diagnosed cases of EPTB (56\%) was not classified based on the body sites involved (Figure2).

\section{Treatment outcomes of PTB and EPTB}

Out of 555 PTB cases put on treatment, 409 have been declared successfully cured, with the overall proportion cured at (73.70\%) and treatment completed at $(17.50 \%)$. Out of 493 EPTB cases put on treatment, 471 have successfully completed treatment (95.53\%) (Table 4).

Cured rate for Pulmonary Bacteriologically

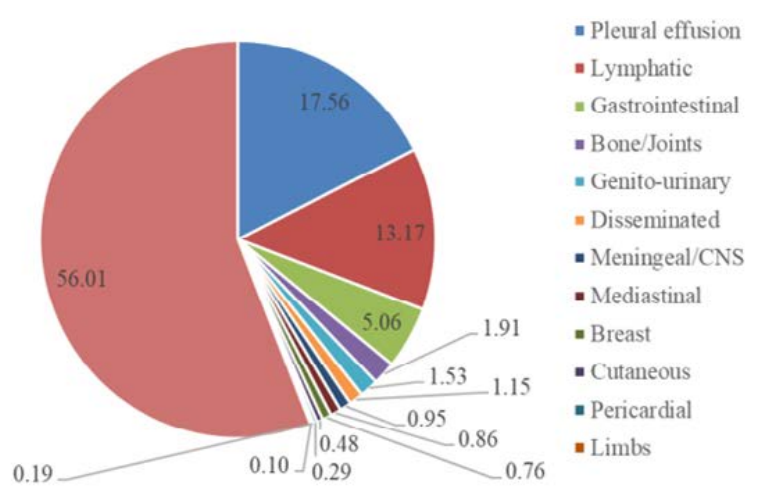

Figure 2. Classification of EPTB by body sites involvement (\%)

Confirmed (PBC) was (91.29\%) (i.e. 409/448*100) and Treatment completion rate for Extrapulmonary Clinically Diagnosed (EPCD) was (95.54\%) (i.e. 471/493*100). TSR for PTB was $(90.97 \%)$ and TSR for EPTB was (95.54\%). The combined TSR of PTB and EPTB was $93.26 \%$ for 2015-2017.

Table 4. Treatment outcome of PTB and EPTB, 2015-2017.

\begin{tabular}{|c|c|c|c|c|c|c|}
\hline \multirow{2}{*}{ Treatment outcome } & \multicolumn{3}{|c|}{ PTB } & \multicolumn{3}{|c|}{ EPTB } \\
\hline & $\mathrm{n}$ & $\%$ & $95 \% \mathrm{Cl}$ & $\mathrm{n}$ & $\%$ & $95 \% \mathrm{Cl}$ \\
\hline Cured & 409 & 73.7 & $70.02-77.37$ & -- & -- & --- \\
\hline Treatment completed & 97 & 17.5 & $14.31-20.65$ & 471 & 95.53 & $93.71-97.37$ \\
\hline Died & 12 & 2.16 & $00.95-03.38$ & 8 & 1.62 & $0.50-2.74$ \\
\hline Treatment failure & 31 & 5.59 & $03.77-07.51$ & 5 & 1.01 & $0.13-1.90$ \\
\hline Lost to follow-up & 4 & 0.72 & $00.00-01.43$ & 5 & 1.01 & $0.13-1.90$ \\
\hline Not evaluated & 2 & 0.36 & $-00.13-08.74$ & 4 & 0.81 & $0.02-1.61$ \\
\hline Cure rate & --- & 91.29 & $88.68-93.91$ & -- & --- & --- \\
\hline Treatment completion rate & --- & 90.65 & $85.14-96.17$ & -- & 95.54 & $93.71-97.37$ \\
\hline Treatment Success Rate (TSR) & --- & 90.97 & $87.03-94.97$ & $--\mid$ & 95.54 & $92.16-99.84$ \\
\hline Overall TSR & \multicolumn{6}{|c|}{93.26 (91.70 - 94.75$)$} \\
\hline
\end{tabular}




\section{DISCUSSION}

In Bhutan, the overall proportion of PTB was $52.95 \%$ while the proportion of EPTB was $47.08 \%$ from 2015-2017. The proportion of EPTB diagnosed appears to be quite high with increasing trend observed till 2016. In 2017, the proportion of EPTB has dropped to about $40 \%$, showing a declining trend. Globally and regionally, researchers have reported that percentage of patients with EPTB ranged anywhere from 15 to $55 \%(7-9)$. It is opinioned that successful treatment and cure of infectious cases may have resulted in a relative increase in annual EPTB case detection ${ }^{(10)}$.

The proportion of clinically diagnosed EPTB in Bhutan from 2015-2017 was 92\%, which is extremely high compared to proportion of bacteriologically diagnosed or smear positive EPTB at $8 \%$, which is very low. Over diagnosis of EPTB is likely, which has led to reporting of a very high percentage of clinically diagnosed EPTB. In a study by Zenebe, Anagaw (11), the overall prevalence of smear positive EPTB was reported as $9.9 \%(34 / 344)$, which is in close proximity to the smear positive EPTB reported in Bhutan.

Diagnosis of EPTB has always been a challenge. EPTB virtually affects all the organs and has a protean clinical manifestation depending on the anatomical sites involved. These often makes diagnosis difficult and presents with dilemma even for clinicians with great deal of experience in the field.

For a definitive diagnosis, it is essential to culture the mycobacteria. An appropriate specimens might be difficult to obtain from extrapulmonary sites due to pauci-bacillary nature of the disease, the bacteriological confirmation of EPTP is often more difficult than for pulmonary tuberculosis. Many of the affected sites may require an invasive procedure to get a biological sample to arrive at the diagnosis. Not all patients may agree for invasive procedures. Even for those who do, due to lack of diagnostic resources, poor yield of conventional diagnostic methods, and the lack of a universal sample processing technique for extra-pulmonary samples, all these can considerably lead to delay in starting the treatment ${ }^{(12)}$.

We report misclassification of miliary $\mathrm{TB} /$ disseminated TB as EPTB in a small percent $(n=12,1.15 \%)$ of our sample. EPTB site was not mentioned in $(56 \%)$ of the patients, which was an important finding of our study. These type of misclassification error can be corrected by following standard diagnostic algorithm and proper documentation of the cases in the TB treatment card and the TB register, both by the treating clinician and the TB In-charges. We also need to sensitize other units (ENT, Surgery, Orthopedics, $\mathrm{MCH}$, General OPD etc.) in the hospitals to refer the patients diagnosed as EPTB for X-ray chest to rule out miliary form of TB or lung involvement, which can avoid such misclassification.

In general, it was found that record keeping in the TB register, TB treatment card and on the TbISS online system was poor and many a times incomplete. We need to revisit all three forms of recording database, update if required and definitely train the new and re-train the existing TB In-charges so that their competencies to upload information present in the TB register onto the TbISS system is enhanced for better compliance and system efficiency in the future.

In terms of diagnostic workload, Jigme Dorji Wangchuk National Referral Hospital in Thimphu has catered to maximum of the TB patients. Thereafter, the patients were either transferred or returned to their place of residence to complete the treatment.

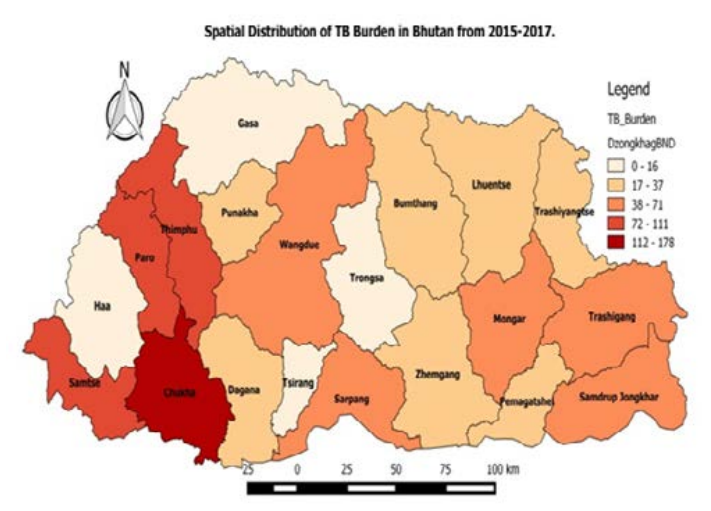

Chukha district followed by Thimphu, Samtse and Paro in the west and Mongar, Trashigang and Samdrupjongkhar in the east had the highest TB cases from 2015-2017 (Figure 3). These are logistic supply, manpower capacity building and programmatic intervention areas by the NTCP.

The combined TSR was $93.26 \%$ from 2015 to 
2017. The overall TSR reported and maintained more than $90 \%$ by the NTCP form thier progarm data has been confirmed and validated through this study.

\section{LIMITATION OF THE STUDY}

As our study was conducted retrospectively, historical data retrieval methods are potential sources of recall biases, missing information and incomplete recording. However, we have collected data by combining all these available sources and validated through rigorous supervision and monitoring process so as to obtain the best available data.

From a total of 31 eligible TB Reporting Centres, we have selected 11 TB reporting centres based on highest cases reported (9 PSUs each for 2015, 2016 \& 2017). In total we had 11 PSUs which means a total of 11 TB reporting sites from nine districts were selected out of 19 eligible districts, representing all regions of the country. Although our sampling plan appears to have resulted in sampling bias, we are confident that results from this study can still be generalized as the TB treatment guidelines, case classification, sputum follow-up examination and treatment outcome standards are followed same throughout the country. On the other hand, mode of diagnostic and investigative procedures such as the sputum microscopy, X-ray and USG are practiced similarly across all centres, but advanced procedures such as the FNAC \& biopsy, MRI \& CT scan, LPA, GeneXperts and culture methods are limited to few centres only. In addition, the total sample size of 1048 retained in the final analysis is statistically robust/powerful enough for generalizability of the findings of the study.

\section{CONCLUSION}

We highly recommend the NTCP to revisit National Guidelines of Management of TB by an expert team on diagnosis of EPTB as very high percentage $(92 \%)$ of EPTB was found to have been diagnosed clinically. Sensitize clinicians and TB In-charges on case detection, to ensure proper case classification, EPTB site identification and proper documentation in TB Register and patient Treatment card. All other units (ENT, Surgery, Orthopedics, MCH, General
OPD etc.) in the hospital should be sensitized on the referral of EPTB cases to Medical Specialist/ Chest Physician for consultation.

\section{CONFLICT OF INTEREST}

None

ACKNOWLEDGEMENT

We would like to express our appreciation and thank the Global Fund for the funding support for this study. We would also like to acknowledge the unwavering support and dedication of the TB In-charges, Health Assistants, Nurses and Office assistants involved in data collection and data entry. We convey our gratitude to the Chief Medical Officers and District Health Officers for facilitating staff release and their participation in this study.

\section{REFERENCES}

1. WHO. Global Tuberculosis Report 2017 Geneva: World Health Organization 2017 [cited 2017 1st November]. Available from: http://www. who.int/tb/publications/global report/high tb burdencountrylists2016-2020.pdf?ua=1.

2. WHO. Tuberculosis Fact Sheet Updated October 2017 Geneva: World Health Organization; 2017 [cited 2017 1st November ]. Available from: http:// www.who.int/mediacentre/factsheets/fs 104/en/.

3. Jamtsho T, Harries A, Malhotra S, Wangchuk D, Dophu U, Dorji T, et al. The burden and treatment outcomes of extra-pulmonary tuberculosis in Bhutan. Public health action. 2013;3(1):38-42.

4. NTCP. Progress Update and Annual Review Meeting 2016-2017. Thimphu: National Tuberculosis Control Programme, 2016.

5. Sharma S, Mohan A. Extrapulmonary tuberculosis. Indian Journal of Medical Research. 2004;120(4):316.

6. MoH. National Guidelines for Management of Tuberculosis Thimphu: Ministry of Health $(\mathrm{MoH})$; 2016. p. 168.

7. Velingker A, Lawande D, Dcosta L. ClinicoEpidemiological Profile of Extra Pulmonary Tuberculosis in Western India. Bone.14:2.83.

8. Mehraj J, Khan ZY, Saeed DK, Shakoor S, Hasan R. Extrapulmonary tuberculosis among females in South Asia-gap analysis. International journal of mycobacteriology. 2016;5(4):392-9. 
9. Purohit M, Mustafa T. Laboratory diagnosis of extra-pulmonary tuberculosis (EPTB) in resourceconstrained setting: state of the art, challenges and the need. Journal of clinical and diagnostic research: JCDR. 2015;9(4):EE01.

10. Arora V, Gupta R. Trends of extra-pulmonary tuberculosis under revised national tuberculosis control programme: A study from South Delhi. Indian Journal of Tuberculosis. 2006;53(2):77.
11. Zenebe $\mathrm{Y}$, Anagaw B, Tesfay W, Debebe T, Gelaw B. Smear positive extra pulmonary tuberculosis disease at University of Gondar Hospital, Northwest Ethiopia. BMC research notes. 2013;6(1):21.

12. Varghese B, Al-Hajoj S. Mapping the epidemiology and trends of extra-pulmonary tuberculosis in Saudi Arabia. International journal of mycobacteriology. 2015;4(4):261-9. 